\title{
Effects of Pore and Grain Size on Water and Polymer Flooding in Micromodels
}

DOI:

10.1021/acs.energyfuels

\section{Document Version}

Accepted author manuscript

Link to publication record in Manchester Research Explorer

\section{Citation for published version (APA):}

Lacey, M., Hollis, C., Oostrom, M., \& Shokri, N. (2017). Effects of Pore and Grain Size on Water and Polymer Flooding in Micromodels. Energy \& Fuels. https://doi.org/10.1021/acs.energyfuels

\section{Published in:}

Energy \& Fuels

\section{Citing this paper}

Please note that where the full-text provided on Manchester Research Explorer is the Author Accepted Manuscript or Proof version this may differ from the final Published version. If citing, it is advised that you check and use the publisher's definitive version.

\section{General rights}

Copyright and moral rights for the publications made accessible in the Research Explorer are retained by the authors and/or other copyright owners and it is a condition of accessing publications that users recognise and abide by the legal requirements associated with these rights.

\section{Takedown policy}

If you believe that this document breaches copyright please refer to the University of Manchester's Takedown Procedures [http://man.ac.uk/04Y6Bo] or contact uml.scholarlycommunications@manchester.ac.uk providing relevant details, so we can investigate your claim.

\section{OPEN ACCESS}




\title{
1. Effects of Pore and Grain Size on Water and Polymer Flooding in Micromodels
}

\author{
${ }_{3}$ Mike Lacey, ${ }^{\dagger}$ Cathy Hollis, ${ }^{\dagger}$ Mart Oostrom, ${ }^{\ddagger}$ and Nima Shokri*, ${ }^{\S}$ \\ $4{ }^{\dagger}$ School of Earth, Atmospheric and Environmental Science, University of Manchester, Manchester, U.K. \\ 5 INTERA Incorporated, Richland, Washington 99354, United States \\ ${ }^{\S}{ }^{\S}$ School of Chemical Engineering and Analytical Science, University of Manchester, Manchester, U.K.
}

7 S Supporting Information

8 ABSTRACT: We have conducted a comprehensive series of experiments to evaluate the effects of pore size distribution of 9 porous media on the dynamics of shear-thinning fluid flow and oil displacement efficiency. To do so, we have conducted 10 microfluidic experiments, using micromodels fabricated from X-ray computed tomography images of sand-packs with varying 11 grain sizes to create a realistic pore network. Three micromodels with well-defined particle size distribution were fabricated and 12 used in our experiments. The use of micromodels to assess the effectiveness of polymer flooding is often superior to methods 13 such as core flooding because of the detailed pore-scale information obtained during the experiments. The micromodels were 14 initially saturated by oil, and the displacing fluids were prepared as aqueous solutions with dissolved xanthan gum. The dynamics 15 and patterns of the interface displacement as well as the size distribution of the trapped oil ganglia were visualized using an 16 optical microscope. The main findings of these experiments are that polymer flooding produces higher recoveries compared to 17 water flooding. However, the effect of pore size on oil entrapment is not uniform across different viscosity ratios, and the 18 relationship between particle size and the quantity of trapped oil ganglia is not consistent across the pore size ranges of the 19 micromodels. Polymer flooding is less efficient in systems with smaller pores, where capillary forces dominate over viscous forces. 20 These findings extend our understanding of the mechanisms controlling the effectiveness of polymer flooding for oil recovery.

\section{INTRODUCTION}

21 Oil recovery from conventional subsurface hydrocarbon 22 reservoirs is notoriously low with, on average, more than 23 two-thirds of oil being unrecovered. ${ }^{1,2}$ There are several levers 24 which impact field-wide recovery factor in conventional oil 25 reservoirs, including pore-scale displacement, sweep, drainage 26 (i.e., areas of the reservoir efficiently drained by the production 27 wells), and economic cut-offs (i.e., the length of time the field 28 can continue producing before economic loss occurs.). ${ }^{3-5}$ The 29 total recovery factor can be increased by extracting more oil by 30 improving total recovery due to any one of these four levers. 31 The areas focused on in this work are pore scale displacement 32 and sweep. One of the most common techniques used for 33 improved reservoir sweep is waterflooding, which can isolate 34 pockets of oil as they are bypassed by the water, splitting the oil 35 into continuous and discontinuous phases in the swept or 36 unswept areas of the reservoir, respectively. ${ }^{1,6}$

37 The effectiveness of the makeup of the injecting fluid on 38 improving sweep efficiency has been the main focus of much 39 research, with many studies conducted into the different 40 properties which alter the final recovery. ${ }^{7-14}$ The use of shear41 thinning fluids such as polymers and foams has been the focus 42 of much recent work ${ }^{8,9,11,13,14}$ both from an experimental and a 43 computational fluid dynamics (CFD) standpoint. It has been 44 shown in numerous studies that the addition of polymers 45 increases recovery ${ }^{11,15}$ due primarily to the associated increase 46 in viscosity.

47 Microfluidic analysis provides an excellent opportunity to 48 look into the details of pore scale displacement processes 49 during oil displacement in porous media at a high resolution (where pores of a few millimeters diameter can be easily 50 visualized). The technology has applications across science and 51 engineering and has advanced greatly in recent years, with 52 improvements in fabrication meaning that models can with- 53 stand higher pressures and create more realistic geological pore 54 geometries. ${ }^{16}$ Furthermore, shorter experiment lengths, lower 55 reagent volumes, and low costs, compared to larger-scale 56 experiments, mean that the use of microfluidic devices is 57 attractive to both industry and academia. The use of 58 micromodels to assess polymer effectiveness is often superior 59 to common methods such as core flooding and homogeneous 60 modeling when studying pore-scale phenomena because it is 61 cheaper and often more representative of the medium than 62 modeling. There are limitations to be aware of when using 63 microfluidics, which are a result of the two-dimensional (2D) 64 nature of the experimental setup. The fact that the depth of the 65 micromodel is constant means that phenomena such as snap-off 66 cannot be accurately studied (Conn et al. ${ }^{17}$ ). A further 67 limitation is due to the uncertainty attached to studying a 68 limited number of porous media. This uncertainty can be 69 reduced using stochastic modeling, as shown by Tahmahsebi et 70 al. $^{18,19}$

Meybodi et al. ${ }^{14}$ conducted a number of waterflood and 72 polymer flood experiments using homogeneous glass micro- 73 models under different wettability conditions and different 74 molecular weights of polymer, degrees of polymer hydrolysis, 75

Received: May 2, 2017

Revised: July 31, 2017

Published: August 4, 2017 
76 and polymer concentration. In the strongly water wet medium, 77 they observed connate water in thick films on the pore walls, 78 whereas in the strongly oil wet medium, a trapped water phase 79 was observed within the oil. Additionally, they illustrated that a 80 higher polymer molecular weight, concentration, and degree of 81 hydrolysis results in a higher apparent viscosity for polymer 82 solution and lower oil-polymer viscosity ratio. This influences 83 the ultimate oil recovery. The effects of capillary and viscous 84 forces affecting the immiscible displacement in porous media 85 were investigated by Rodriguez De Castro et al. ${ }^{11}$ They used 86 the capillary number and viscosity ratio between two fluid 87 phases to quantify the strength of the capillary and viscous 88 forces within the micromodel. Throughout their experiments 89 they used fluids with varying polymer concentrations to 90 displace oil within a hydrophilic micromodel. The results 91 clearly show that the inclusion of polymer in the displacing fluid 92 increases oil recovery at all concentrations relative to water, 93 with the lowest residual oil occurring at the highest polymer 94 concentration for all flow rates. It is also worth noting that 95 residual oil saturation decreases with increasing polymer 96 concentration at each capillary number that was assessed. 97 Finally, a range of polymer compositions has been investigated 98 by Buchgraber et al. ${ }^{15}$ in order to compare the current standard 99 composition with newly formulated polymers. Although 100 different polymers exhibited visual differences in the displace101 ment front stability, there was no difference in final recovery. 102 Much work has been carried out on the effect of the fluid 103 rheology on pore scale recovery; ${ }^{8-14}$ there has been little work 104 to characterize the effect of the topology of the porous medium 105 itself on total recovery efficiency. Although the pore geo106 metrical controls on permeability have been widely discussed in 107 the geological literature,,$^{20,21}$ focus has been on single-phase 108 fluid flow. In addition, the majority of the previous microfluidic 109 analyses on the performance of polymer flooding was 110 conducted in relatively homogeneous $2 \mathrm{D}$ porous media. The 111 use of micromodels for experimental analysis has recently been 112 extended from simple geometric design to more complicated 113 designs based on real rock fabrics, using thin section and 114 computed tomography images. For example, Nilsson et al. ${ }^{2}$ 115 developed a microfluidic system based on sandstone to enable 116 the rapid assessment of the suitability of a certain polymer for 117 differing oil reservoir scenarios. In this work we describe water 118 and polymer flooding displacement experiments in three 119 micromodels to quantitatively investigate the effects of grain 120 and pore size on residual oil saturation and oil ganglia size 121 distribution. The micromodels were designed and fabricated 122 using three-dimensional (3D) X-ray images of actual sand packs 123 to provide a more realistic representation of geological 124 heterogeneity.

\section{MATERIALS AND METHODS}

125 2.1. Micromodels. This study uses three distinct micromodels 126 with different pore networks (Figure 1). The models were fabricated 127 based on X-ray CT images of sand packs. ${ }^{22}$ Each model is $16 \mathrm{~mm}$ long 128 by $8 \mathrm{~mm}$ wide, with a depth of $39.9 \pm 1 \mu \mathrm{m}$, with the field of view 129 visualized in these experiments being 12.7 by $9.5 \mathrm{~mm}$, due to imaging 130 size constraints. The physical descriptions of the porous media are 131 presented in Table 1 . The average particle size of each media was 132 determined using a laser diffraction particle size analyzer. ${ }^{22}$ Although 133 the three micromodels have distinct grain size ranges, the coarse- and 134 medium-grained models have approximately the same average pore 135 sizes of 0.31 and $0.32 \mathrm{~mm}$, respectively, allowing the effects of grain 136 size on oil recovery efficiency to be investigated for systems with 137 different degrees of grain size homogeneity. The sorting of the grains
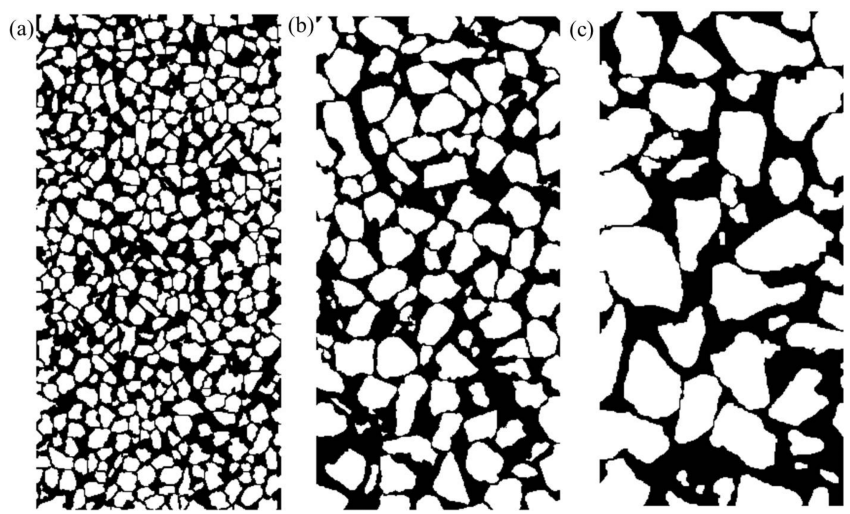

Figure 1. Patterns of micromodels used in the present study. Black and white represents pores and grains, respectively. Panels $a, b$, and $c$ represent fine-, medium-, and coarse-grained porous medium, respectively. The micromodels are $16 \mathrm{~mm}$ long and $8 \mathrm{~mm}$ wide.

Table 1. Micromodel Characteristics (Adapted from Norouzi Rad et al. ${ }^{22}$ ) with the Sorting Calculated Based on the Variance of the Grain Sizes within the Micromodel Using the Udden-Wentworth Phi Scale

\begin{tabular}{llll} 
& \multicolumn{3}{c}{ micromodel } \\
\cline { 2 - 4 } \multicolumn{1}{c}{$\begin{array}{c}\text { porous medium } \\
\text { characteristic }\end{array}$} & \multicolumn{1}{c}{ fine } & medium & coarse \\
average Grain Size $(\mathrm{mm})$ & 0.6 & 1.4 & 2.1 \\
grain Size Range $(\mathrm{mm})$ & $0.36-0.77$ & $0.2-1.6$ & $1.3-2.7$ \\
sorting (-) & 1.16 & 1.81 & 2.39 \\
porosity $(\%)$ & 49.64 & 42.27 & 41.87 \\
average pore size $(\mathrm{mm})$ & 0.16 & 0.32 & 0.31 \\
pore volume $\left(\mathrm{mm}^{3}\right)$ & 2.5 & 2.2 & 2.1 \\
\hline
\end{tabular}

(a measure of the variance in grain sizes) within the micromodel is 138 linearly related to the average grain size of each micromodel. The sand 139 packs used for the micromodels comprise sandstones that have a very 140 coarse, coarse, or medium-coarse grain size according to the Udden- 141 Wentworth grain size scale commonly used to describe clastic rocks. ${ }^{23} 142$ For the purposes of this project we refer to them relative to each other 143 as coarse-, medium-, and fine-grained.

The micromodels were fabricated in a silicon wafer using standard 145 photolithography and inductively coupled plasma-deep reactive ion 146 etching (ICP-DRIE) methods. A detailed description of the process 147 can be found in Willingham et al. ${ }^{24}$ To enable fluid flow through the 148 model, nanoports were incorporated into the inlet and outlet 149 chambers of the micromodels. Finally, the micromodels were 150 chemically treated to obtain uniform wettability. At the end of this 151 process, the micromodels demonstrated a stable and uniform 152 hydrophilic behavior with air-water contact angles $<16^{\circ} .^{21}$ The effect 153 of wettability is not studied in this work, but it has been investigated by 154 Rabbani et al. ${ }^{25}$

2.2. Fluid Properties. There are four fluid pairs used in this study, 156 consisting of combinations of two displacing fluids and two defending 157 fluids. The displacing fluids were deionized water and a 1000 ppm 158 xanthan gum polymer solution. The defending fluids were Xiameter 159 PMX - 200 Silicon Fluid (Dow Corning, Midland, Michigan) and 160 Exxsol D140 (Exxon Mobil, Irving, Texas). The deionized water, 161 polymer solution, silicon fluid, and the Exxsol oil, are referred to as W, 162 P, O1, and O2, respectively. Pertinent fluid properties of the four fluids 163 are listed in Table 2. The polymer solution is a shear-thinning fluid, as 164 t2 shown by Rodríguez De Castro et al. ${ }^{11}$ The addition of polymer did 165 not significantly alter the interfacial tension at the concentration of 166 polymers used in our experiments.

2.3. Experimental Procedure. The experimental setup is shown 168 in the Supporting Information. Displacement experiments were 169 conducted in the three micromodels for all four fluid pairs listed in $170 \mathrm{t} 3$ 
Table 2. Viscosity and Interfacial Tensions of the Fluids Used in Our Experiments

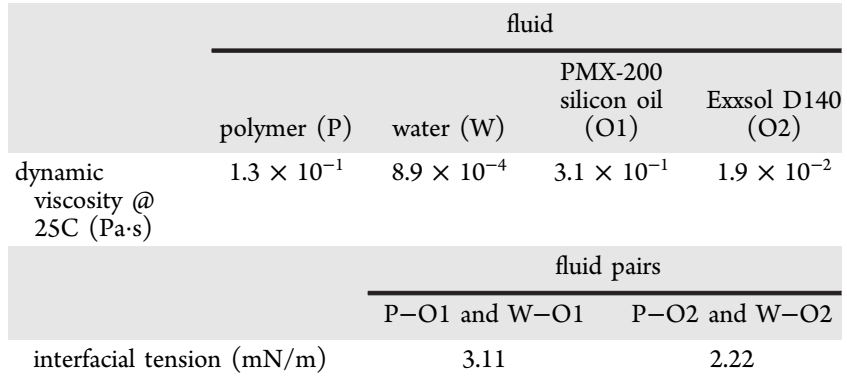

171 Table 3. For each of the 12 micromodel-fluid pair combinations, the 172 experiment was conducted three times resulting in a total of 36 rounds

Table 3. Viscosity Ratios (M) and Capillary Numbers $(\mathrm{Ca})$ for the Four Fluid Pairs ${ }^{a}$

\begin{tabular}{lcccc} 
& \multicolumn{4}{c}{ fluid pairs } \\
\cline { 2 - 5 } & $\mathrm{P}-\mathrm{O} 1$ & $\mathrm{P}-\mathrm{O} 2$ & $\mathrm{~W}-\mathrm{O} 1$ & $\mathrm{~W}-\mathrm{O} 2$ \\
viscosity ratio, $\log M$ & -0.38 & 0.84 & -2.5 & -1.3 \\
capillary number, $\log \mathrm{Ca}$ & -2.4 & -2.3 & -4.6 & -4.5
\end{tabular}

${ }^{a}$ The displacement velocity for all experiments is $8.7 \times 10^{-4} \mathrm{~m} / \mathrm{s}$.

173 of experiments. The three experiments conducted with each fluid pair 174 and micromodel combination are termed an "experimental series" in 175 the remainder of the paper.

176 During each experiment, the model was initially saturated with one 177 of the two oils at a flow rate of $100 \mathrm{~mL} /$ hour. After saturation, an 178 initial image was captured as a reference for subsequent image analysis. 179 The oil was then displaced by injecting either deionized water, dyed 180 with 1 w/w\% Ecoline 100 dye (Royal Talens, The Netherlands), or the dyed xanthan solution at a constant flow rate of $1 \mathrm{~mL} /$ hour using a 181 syringe pump (PHD Ultra 3000, Harvard Apparatus), and images of 182 the displacement were captured at one second intervals. The injected 183 rate resulted in an average pore water velocity of $8.7 \times 10^{-4} \mathrm{~m} / \mathrm{s}$. After 184 steady state had been obtained (i.e., the phase saturation distributions 185 remain constant), a final image of the phase distribution was captured 186 before the flow was stopped.

Prior to each experimental series, the model was restored to its 188 original condition by carrying out the following cleaning procedure. A 189 series of five rinses were conducted in the following order: deionized 190 water, isopropanol, deionized water, SC-1, and deionized water, where 191 SC-1 stands for step 1 of the Standard Clean described by Kern. ${ }^{26}$ SC- 192 1 was carried out with a solution made up of 5 parts deionized water, 1193 part aqueous $\mathrm{NH}_{4} \mathrm{OH}\left(29\right.$ wt \%), and 1 part aqueous $\mathrm{H}_{2} \mathrm{O}_{2}$ (30 wt \%). 194 More than 100 pore volumes were injected at $100 \mathrm{~mL} / \mathrm{h}$ during each 195 of the 5 steps. After cleaning, the model was dried using pressurized air 196 (1500 mbar) using a pressure generator (Elveflow, France). For each 197 repetition within an experimental series, the model was cleaned using 198 the standard simplified cleaning procedure in our lab comprising three 199 rinses: deionized water, isopropanol, and deionized water, followed by 200 drying using pressurized air.

The viscosity ratio $(M)$ and capillary number $(\mathrm{Ca})$ for the four 202 displacement experiments are listed in Table 3. The two dimensionless 203 numbers are defined as follows:

$$
\begin{gathered}
M=\frac{\mu_{\mathrm{i}}}{\mu_{\mathrm{d}}} \\
C a=\frac{\mu_{\mathrm{i}} v}{\gamma}
\end{gathered}
$$

where $\mu_{\mathrm{i}}$ (Pa s) is the dynamic viscosity of the invading fluid, $\mu_{\mathrm{d}}$ (Pa s) 207 the dynamic viscosity of the defending fluid, $v(\mathrm{~m} / \mathrm{s})$ the velocity of 208 the invading fluid, and $\gamma(\mathrm{N} / \mathrm{m})$ the interfacial tension between the 209 invading and defending fluids.

As shown in Table 3, with a range in $M$ and $C a$ of almost 4 and 2211 orders of magnitude, respectively, several displacement regimes may be 212
Coarse
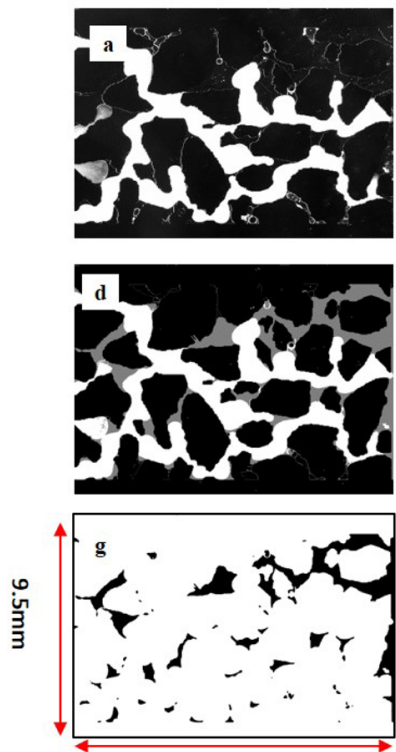

$12.7 \mathrm{~mm}$
Medium
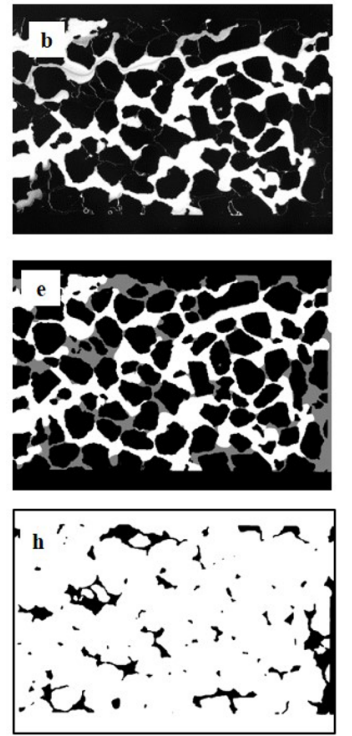

Flow direction

Fine
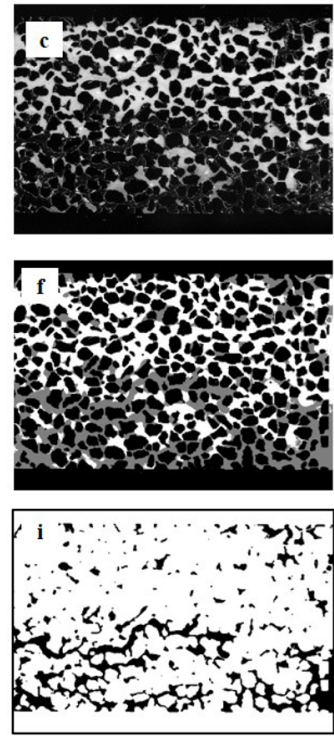

Figure 2. $(a-c)$ Typical examples of the raw images obtained during displacement experiments in fine, medium, and coarse-textured micromodels. $(\mathrm{d}-\mathrm{f})$ Segmented images obtained at the first breakthrough of the displacing fluid (polymer). The displacing fluid in images $\mathrm{d}-\mathrm{f}$ is in white; the grains are black, and the residual oil is gray. $(\mathrm{g}-\mathrm{i})$ Residual oil ganglia derived from images $\mathrm{d}-\mathrm{f}$. Black corresponds to the residual oil phase, and white represents everything else. 

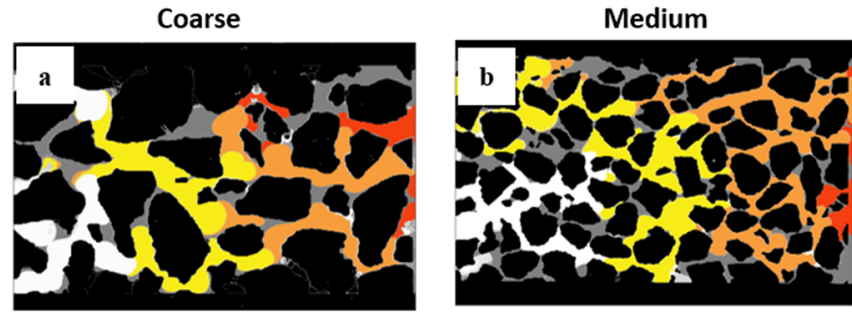

Flow direction
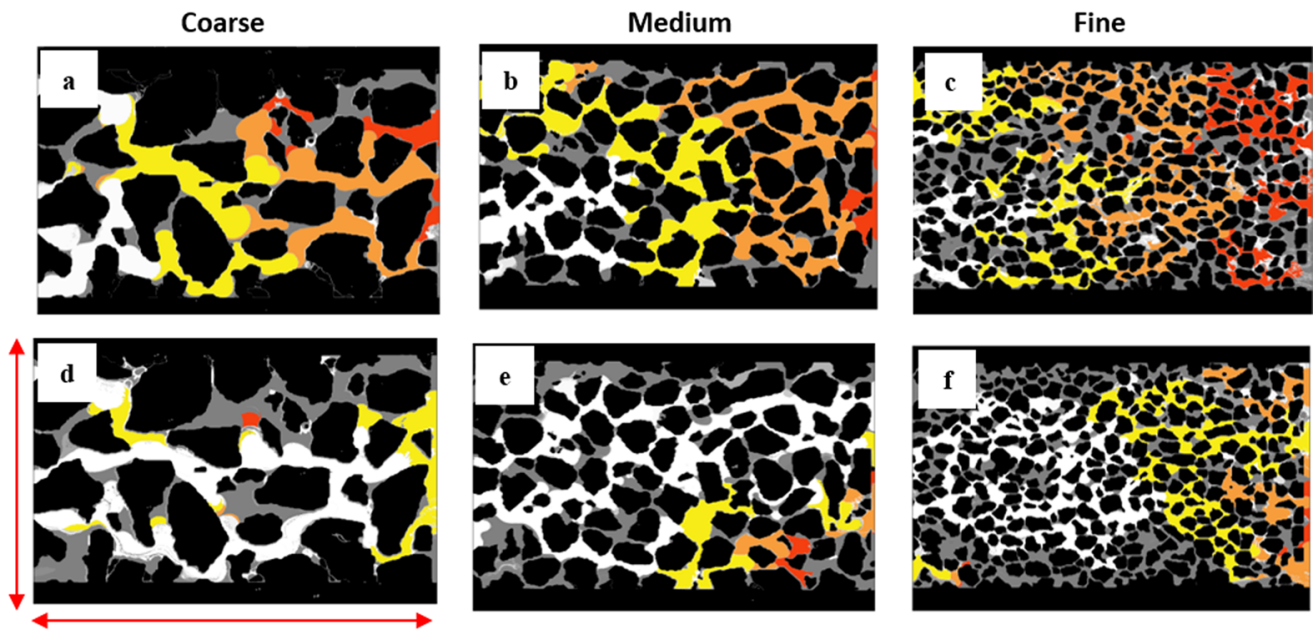

$12.7 \mathrm{~mm}$

Figure 3. Fluid saturations in the micromodel at times $t, t+\Delta t, t+2 \Delta t$, and $t+3 \Delta t$, where $\Delta t$ is $2 \mathrm{~s}$. Panels a-c denote displacement $\mathrm{P}-\mathrm{O} 2$, while panels $\mathrm{d}-\mathrm{f}$ denote displacement $\mathrm{W}-\mathrm{O} 2$. The dark areas represent grains. White, yellow, orange, and red represent polymer $(\mathrm{a}-\mathrm{c})$ and water $(\mathrm{d}-\mathrm{f})$ saturations at different timesteps throughout the experiment $(t, t+\Delta t, t+2 \Delta t$, and $t+3 \Delta t$, respectively). Gray represents the pore spaces filled by residual oil at time $t+3 \Delta t$.

213 expected, $^{27-29}$ including capillary fingering, viscous fingering, and 214 crossover.

215 2.4. Image Analysis. The dynamics of oil displacement and 216 residual oil ganglia distribution were visualized using an optical 217 microscope (Leica M205C, 20.5:1 zoom, maximum resolution of $2180.925 \mu \mathrm{m}$, equipped with a Leica DFC 3000G high resolution digital 219 camera). The images were analyzed using a combination of MATLAB 220 and ImageJ. The images were segmented using a custom-designed 221 MATLAB code. The segmentation process consisted of four sequential 222 steps: (1) A reference image was captured where the entire pore space 223 was flooded with white dyed liquid, allowing a segmentation mask for 224 the fluids in the pore space to be created. (2) The images were filtered 225 using a median filter. (3) The mask was used to isolate the pore space 226 in the experimental images, with the background labeled as grains and 227 assigned a grayscale value of 0 in the output image.(4) The fluid 228 images were segmented within the pore space, using a static greyscale 229 threshold determined by assessing the greyscale histogram and 230 choosing the point equidistant from the two major peaks. Overall, a 231 good agreement between the fluid distribution in the original image 232 and the segmented image was achieved. Figure 2 illustrates typical 233 examples of the raw images recorded by the microscope; the 234 segmented images distinguishing between oil, displacing fluid, and 235 matrix; and the location of the residual oil phase at the first 236 breakthrough of the displacing fluid.

237 In order to minimize micromodel boundary effects, a window with 238 the size $12.7 \mathrm{~mm} \times 6.6 \mathrm{~mm}$ was selected for quantification. This size 239 was selected after evaluations of different window sizes to ensure that 240 the selected area was representative of the residual oil and blob size 241 distribution for the whole model. The distribution and morphology of 242 residual oil ganglia were visualized and quantified using ImageJ. 243 Following segmentation using MATLAB, images such as those 244 presented in Figure $2 \mathrm{~d}-\mathrm{f}$ were then thresholded in ImageJ to reduce 245 the image to 2 phases-the residual oil phase and everything else (i.e 246 the flooded area and grains), as shown in Figure $2 \mathrm{~g}-\mathrm{i}$. The Image 247 "Analyse particle function" was then used to obtain the quantity and 248 morphology of the residual oil ganglia. Two particular times of interest 249 in our analysis are the breakthrough time $\left(T_{b}\right)$ and the time to final 250 conditions $\left(T_{\mathrm{f}}\right)$. The $T_{\mathrm{b}}$ is defined as the earliest time at which the 251 injecting fluid reaches the micromodel exit, and $T_{\mathrm{f}}$ is defined as the 252 point where steady state was reached and no more oil is produced by 253 the displacing aqueous phase (i.e., the phase saturation distribution 254 remains constant throughout the micromodel). The residual oil saturations at $T_{\mathrm{b}}$ and $T_{\mathrm{f}}$ were calculated in MATLAB using the 255 segmented images.

\section{RESULTS AND DISCUSSION}

3.1. Oil Recovery. Analysis of the segmented images, taken 257 at one second intervals during the experiment, allowed 258 visualization of the dynamics of the advancing displacement 259 front. Figure 3 shows the displacement front at $2 \mathrm{~s}$ intervals for $260 \mathrm{f} 3$ the $\mathrm{W}-\mathrm{O} 2$ and $\mathrm{P}-\mathrm{O} 2$ displacements. This qualitative visual- 261 ization shows that the polymer displacement front progresses 262 more slowly and in a more stable manner than the water 263 displacement front in all three micromodels, although the effect 264 appears more pronounced in the coarse- and medium-grained 265 models. The figure suggests that the volume of oil displaced by 266 polymer solution in each time step is similar, particularly in the 267 medium- and coarse-grained models.

The polymer solution achieves higher recovery efficiency 269 than water at both $T_{\mathrm{b}}$ and $T_{\mathrm{f}}$ for each comparable experimental 270 series, i.e., $\mathrm{P}-\mathrm{O} 1$ achieves higher recovery $(65 \%)$ at $T_{\mathrm{f}}$ than 271 $\mathrm{W}-\mathrm{O} 1(55 \%)$ in the fine-grained micromodel, except in the 272 case of $\mathrm{P}-\mathrm{O} 2$ at $T_{\mathrm{f}}$ in the coarse-grained micromodel. Figure $4 \mathrm{a} 273 \mathrm{f} 4$ shows that at $T_{\mathrm{b}}$, the recovery is the highest for the coarse- 274 grained micromodel in two of the 4 cases, where oil 2 is used. 275 For the $\mathrm{P}-\mathrm{O} 1$ displacement the trend is for the recovery to 276 decrease with increasing grain size, while $\mathrm{W}-\mathrm{O} 1$ shows no clear 277 trend with increasing grain size.

278

At $T_{\mathrm{f}}$, the recovery is not affected by the particle size for three 279 of the four displacements (Figure 4b). Only for the P-O2 fluid 280 pair is the oil recovery smaller in the fine-grained micromodels 281 compared to the other two. As shown in Table 1, these grain 282 size categories equate to different average pore sizes with sizes 283 of $0.6,1.4$, and $2.1 \mathrm{~mm}$ being equivalent to average pore sizes of 284 $0.16,0.32$, and $0.31 \mathrm{~mm}$, respectively. The final recovery shown 285 in Figure $4 \mathrm{~b}$ for the $\mathrm{P}-\mathrm{O} 2$ fluid pair is therefore clearly higher 286 in the two micromodels with the larger average pore sizes, i.e., 287 the models with average grain sizes 1.4 and $2.1 \mathrm{~mm}$. The largest 288 average pore size (corresponding to average particle size 1.4289 $\mathrm{mm}$ ) also equates to the highest final recovery, while the 290 

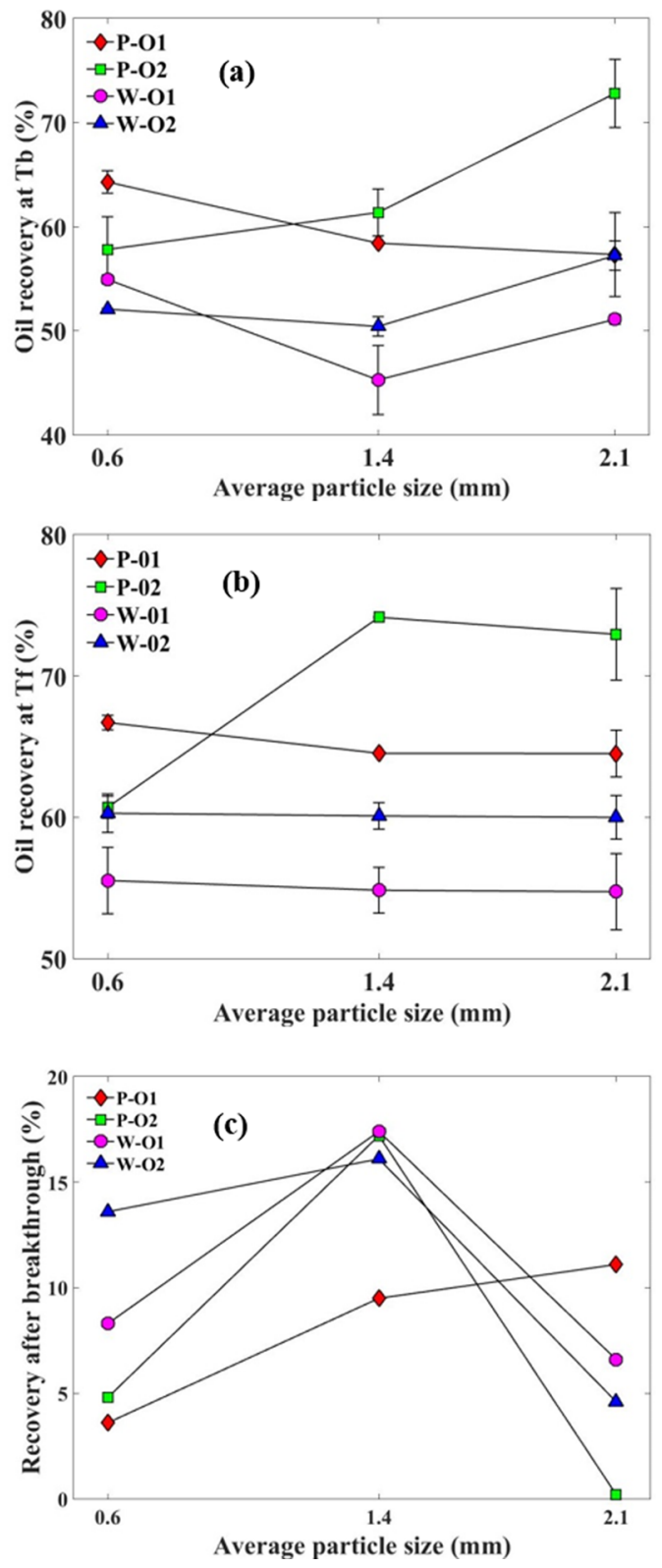

Figure 4. Average oil recovery achieved across three repetitions of experiments at (a) $T_{\mathrm{b}}$ and (b) $T_{\mathrm{f}}$, with error bars showing the standard error. The differences in \% oil recovery at these two times are shown in panel c.

291 micromodel with the smallest average pore size (corresponding 292 to average particle size $0.6 \mathrm{~mm}$ ) has the lowest final recovery. 293 The reason for the different trend in the $\mathrm{P}-\mathrm{O} 2$ case, compared 294 to all other cases is due to the higher viscosity ratio. As the 295 viscosity ratio approaches 1 , the effect of pore size is exhibited; 296 all other fluid pairs have a viscosity ratio sufficiently below 297 parity to negate the effect of differing pore sizes.

298 The polymer solution achieves higher recovery than water at 299 final conditions in almost every case, which is consistent with 300 polymer flooding experiments conducted by Herbas et al. ${ }^{30}$ 301 The dominant variation between the four different fluid pairs is 302 the viscosity ratio. For those fluid pairs with a negative viscosity 303 ratio $(\log M)$, pore size and grain size have minor effecta on 304 final recovery, as shown in Figure 4b. However, for the fluid 305 pair of polymer and oil, where the viscosity ratio approaches 1 306 (P-O1), leading to theoretically more stable displacement, it becomes apparent that there is some control on recovery as a 307 result of pore size, with the medium- and coarse-grained 308 micromodels having similar average pore sizes and residual oil 309 saturations. The distinct change in final recovery between the 310 models with average pore sizes of $0.31,0.32$, and 0.16 suggests 311 that the displacement mechanism differs between the different 312 pore sizes, i.e., moving toward capillary fingering from a more 313 stable displacement (as defined by Lenormand et al. $^{31}$ ).

314

In Figure 4c, the volume of oil recovered postbreakthrough 315 varies significantly across the three micromodels. Prior to 316 breakthrough, the mode of displacement is primarily pistonlike; 317 however, once breakthrough has occurred, the dominant mode 318 of oil displacement changes to snap-off and displaces a portion 319 of the bypassed ganglia, as described by Lenormand et al. ${ }^{27} 320$ Once the mode of displacement changes to snap-off, the effect 321 of pore topology is increased, as the geometry of the grain 322 surfaces affects the critical angle at which snap-off will occur in 323 the defending fluid. The trend observed in Figure 4c suggests 324 that the size and geometries of the grains in the medium- 325 grained model have the most favorable topology for snap-off to 326 occur.

3.2. Displacement Dynamics. For the $\mathrm{P}-\mathrm{O} 1$ and $\mathrm{P}-\mathrm{O} 2328$ fluid pairs ( -0.38 and 0.84 , respectively), which have more 329 favorable $\log M$ values than the $\mathrm{W}-\mathrm{O} 1$ and $\mathrm{W}-\mathrm{O} 2$ pairs 330 $(-2.5$ and -1.3 , respectively), the rate at which oil is displaced 331 is lower as indicated by the slope of the initial portion of the 332 curve in Figure $5 \mathrm{a}-\mathrm{c}$ and the number of pore volumes injected 333 fs to reach the final recovery (Table 4). For the displacements 334 t4 with water, the injected pore volumes needed to reach the final 335 recovery varies between 0.2 and 1 pore volumes. However, for 336 the $\mathrm{P}-\mathrm{O} 1$ and $\mathrm{P}-\mathrm{O} 2$ displacements, the number of pore 337 volumes injected to reach the final residual oil saturation is 338 almost constant with all plots showing asymptotic behavior 339 after injection of approximately 0.8 pore volumes.

340

Figure $6 \mathrm{a}$ shows the volume invaded by the injecting fluid in $341 \mathrm{fo}$ each time step for the $\mathrm{W}-\mathrm{O} 2$ and $\mathrm{P}-\mathrm{O} 2$ displacements in all 342 micromodels, with the same information presented quantita- 343 tively in Figure $6 \mathrm{~b}-\mathrm{d}$. It provides an intriguing insight into the 344 dynamics of the process exhibited by polymer displacement. 345 For the three polymer cases, the volume of oil displaced each 346 second is relatively uniform until the point at which the 347 displacement front reaches the outlet port. In the cases where 348 the pores are larger, i.e., the coarse- and medium-grained 349 models, the volume of oil displaced in each time step is almost 350 identical, as is the time step in which the outlet port is reached. 351 When pore sizes are smaller, the displacement in each time step 352 is larger; therefore, the outlet port is reached earlier. In the case 353 of the fine-grained micromodel, the volumes displaced in each 354 time step for the polymer and water cases are similar. 355

The factor controlling the final recovery in the different 356 micromodels is thought to be the capillary entry pressure, 357 which is higher in the fine-grained micromodel with the smaller 358 average pore size and thinner capillaries. This result suggests 359 that polymer solution is far more effective at increasing the final 360 recovery in reservoir systems where the average pore size is 361 larger, whereas in reservoir systems with narrower pore 362 systems, the addition of polymer into the flooding fluid is 363 less likely to increase total recovery. The reason for this change 364 in displacement mechanism is a change in the dominant force 365 in the flow, i.e., the flow changes from a more viscous 366 dominated flow to a capillary dominated flow. The volume 367 displaced by polymer solution in each time step until $T_{\mathrm{b}}$ in 368 Figure 6a is almost identical. This alongside the fact that the 369 

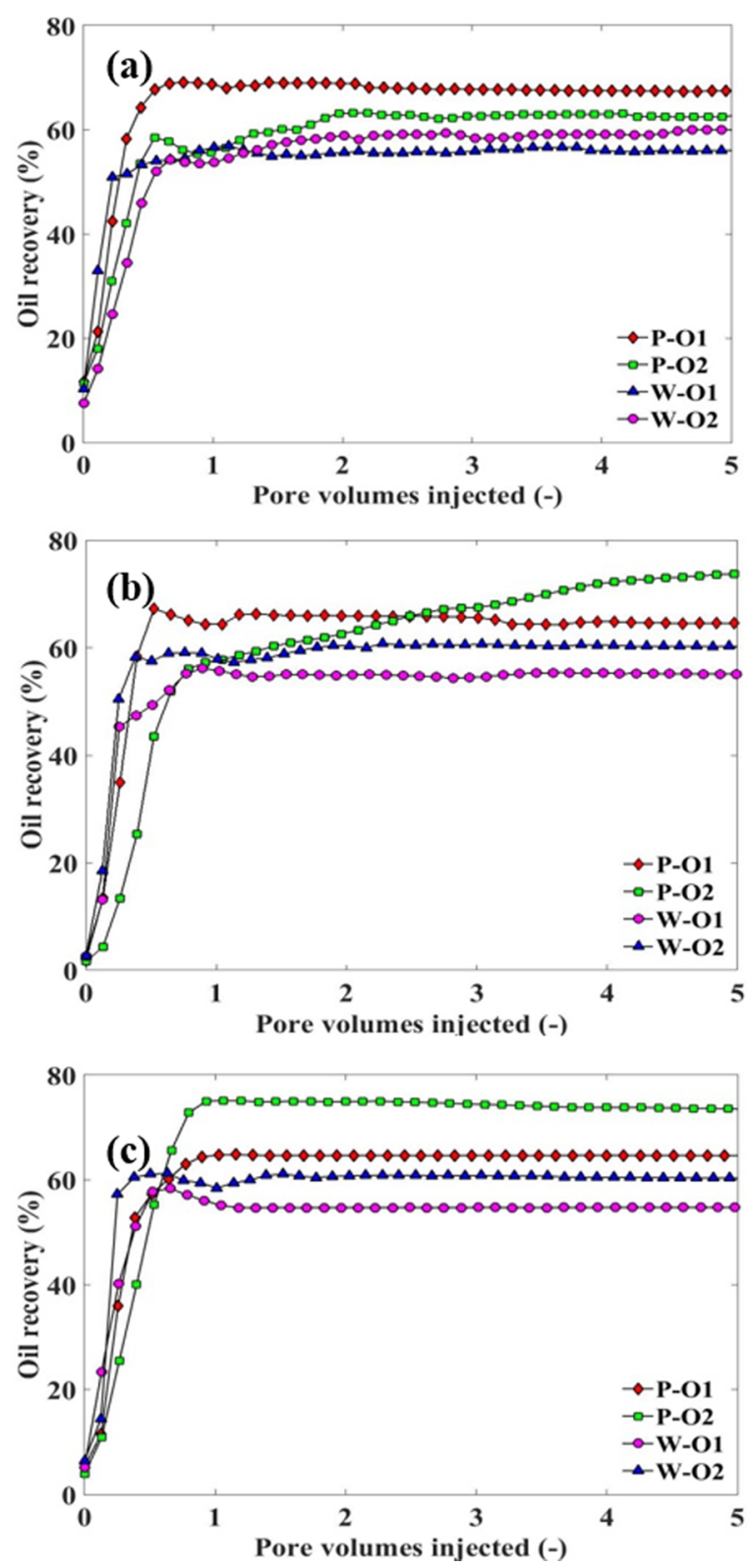

Figure 5. Oil recovered as a function of pore volumes injected for (a) fine-, (b) medium-, and (c) coarse-grained micromodels.

Table 4. Initial Gradient for Each of the Curves Presented in Figure 5

\begin{tabular}{llcc} 
& fine & medium & coarse \\
$\mathrm{P}-\mathrm{O} 1$ & 1.1 & 1.34 & 1.12 \\
$\mathrm{P}-\mathrm{O} 2$ & 0.92 & 0.78 & 0.91 \\
$\mathrm{~W}-\mathrm{O} 1$ & 1.81 & 1.67 & 1.17 \\
$\mathrm{~W}-\mathrm{O} 2$ & 0.84 & 1.57 & 2 \\
\hline
\end{tabular}

370 recovery efficiency for the $\mathrm{P}-\mathrm{O} 2$ fluid pair shown in Figure 4 is 371 almost identical to that of waterflooding demonstrates that 372 polymer solution may have less potential for improving 373 recovery of certain oil types in systems where the pores are 374 smaller and therefore the capillary forces are more dominant. 375 The smallest average pore size in these micromodels 376 corresponds to the smallest grain size $(0.6 \mathrm{~mm})$, which is 377 equivalent to a medium-coarse grained sandstone on the 378 Udden-Wentworth grain size classification. This means that polymer solution may not improve recovery in a large number 379 of reservoir sandstones, which are typically fine-medium 380 grained, if grain size is assumed to be a proxy for pore size, 381 which is a reasonable assumption in many sandstones. ${ }^{32} 382$

The number of pore volumes injected at the point where 383 final recovery is reached for all polymer-oil fluid pairs is much 384 more similar between the three models than for water-oil fluid 385 pairs, as shown in Figure 5. This analysis coupled with the 386 visualizations provided in Figures 3 and 6 show that, at this 387 scale, the addition of polymer to the displacing fluid leads to a 388 far more stable displacement front in cases where the pores are 389 greater than that of the fine-grained micromodel. The fact that 390 only two distinct pore sizes have been investigated in this work 391 means that it is not possible to clearly resolve the point at 392 which the polymer becomes more effective than water flooding. 393 The transition between capillary-dominated flow and viscous- 394 dominated flow with changing pore size is likely to be a gradual 395 transition, with a zone of flow where neither force is truly 396 dominant.

397

3.3. Oil Entrapment. Oil entrapment during displacement 398 is a critical constraint on hydrocarbon production, because 399 bypassed oil is very difficult to produce once the flooding front 400 has passed by it. The term bypassed oil, for the purpose of this 401 work, are the volumes of oil not produced in a region which has 402 been passed by the displacement front. Figure 7 shows the $403 \mathrm{f} 7$ percentage of the total oil ganglia in the micromodels which 404 have a certain size. The size it shows is defined as the number 405 of average sized pores filled, using the average pore sizes 406 defined earlier for each of the micromodels.

The addition of polymer reduces the size of the largest 408 trapped oil ganglion (Figure 7a,b). For these cases, ganglia are 409 generally considerably smaller than those for water displace- 410 ments (Figure $7 c, d$ ). In our experiments, we did not find a clear 411 trend in the relationship between pore/grain size and the 412 percentage of ganglia of differing sizes. Additionally, the 413 number of bypassed oil ganglia in each of the micromodels 414 for each fluid pair at $T_{\mathrm{f}}$ was determined using the Image 415 software (Figure 8). The results indicate a general decreasing $416 \mathrm{f} 8$ trend between grain size and number of residual oil ganglia. 417 Although not all of the fluid pairs show a linear relationship, the 418 general trend is that as average particle size increases, the 419 number of ganglia decreases. Such a relationship may also be 420 dependent on viscosity ratio.

The use of polymer as the injecting fluid led to a reduction in 422 the size of the largest oil ganglion in the majority of cases. This 423 is because the more displacement was more stable in the cases 424 where polymer is the displacing fluid. This more pistonlike 425 displacement means that the oil in the model is more 426 homogeneously displaced, leading to fewer large ganglia being 427 bypassed. The size of the residual ganglia within the 428 micromodels fits well with those reported by others, ${ }^{33}$ with 429 the majority being between 1 and 15 pore volumes. The 430 number of ganglia in each of the different micromodels is 431 dependent on the particle size and viscosity ratio. It is apparent 432 that there appears to be a relationship between viscosity ratio 433 and the linearity of the change in ganglia numbers with 434 increasing grain size. Further experimental data would be 435 necessary to fully discern the nature of this relationship; 436 however, from these results it is thought that in the cases where 437 the viscosity ratios are lower, the number of ganglia is more 438 dependent on the fluid. Conversely, when the viscosity ratio is 439 higher, and the displacement front is therefore more stable, the 440 


\begin{tabular}{|c|c|c|c|c|c|c|c|c|c|}
\hline Time (s) & 1 & 2 & 3 & 4 & 5 & 6 & & 7 & 8 \\
\hline $\begin{array}{l}\mathrm{P}-\mathrm{O} 2 \\
\text { Fine }\end{array}$ & $\frac{\pi}{2}$ & " & 然然 & 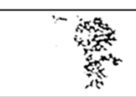 & $\therefore \frac{5}{4}$ & $=\quad-$ & & $\therefore$ & $\hat{r}$ \\
\hline $\begin{array}{l}\mathrm{W}-\mathrm{O} 2 \\
\text { Fine }\end{array}$ & 籍好 & 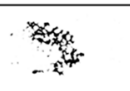 & 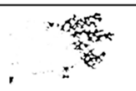 & 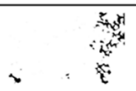 &.$\quad \quad \quad r$ & : & & $\therefore$ & . \\
\hline $\begin{array}{l}\mathrm{P}-\mathrm{O} 2 \\
\text { Medium }\end{array}$ & 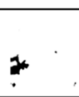 & the & 洝 & res & "rest & & & 7 & \\
\hline $\begin{array}{l}\mathrm{W}-\mathrm{O} 2 \\
\text { Medium }\end{array}$ & 次 & . & ${ }^{\prime}{ }^{2}$ & 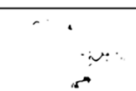 & 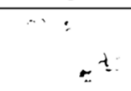 & 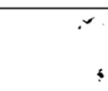 & & $\therefore$ & $?$ \\
\hline $\begin{array}{l}\mathrm{P}-\mathrm{O} 2 \\
\text { Coarse }\end{array}$ & $j$ & $\vec{x}$ & . & 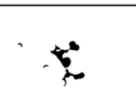 & مa & $\therefore$ & & $\therefore \vec{i}$ & $\therefore 4$ \\
\hline $\begin{array}{l}\mathrm{W}-\mathrm{O} 2 \\
\text { Coarse }\end{array}$ & 2 & is & $i$ & $\therefore$ & $\therefore$ & . & & $\begin{array}{c}- \\
-\quad \therefore\end{array}$ & Ji \\
\hline
\end{tabular}
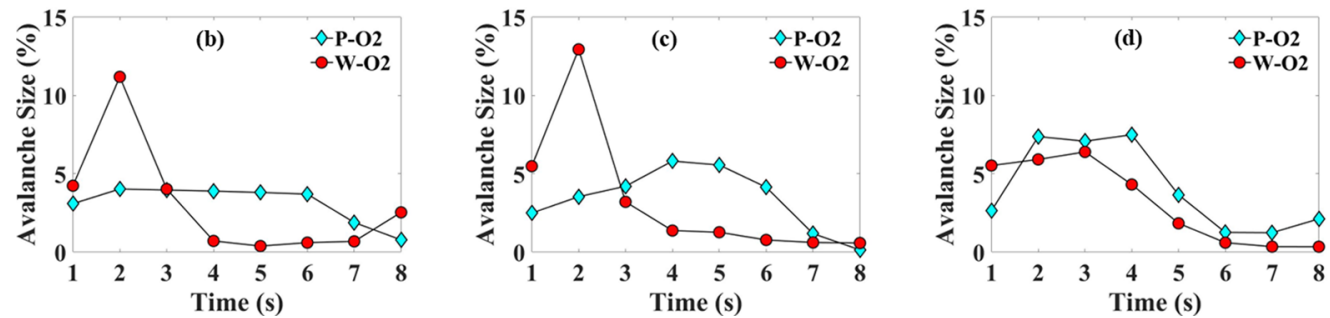

Figure 6. (a) Volume invaded in each of the first eight time steps shown visually for polymer and water displacing oil 2, with black showing the displaced fluid. $(b-d)$ Volume invaded in each of the first eight time steps shown graphically for polymer and water displacing oil 2.
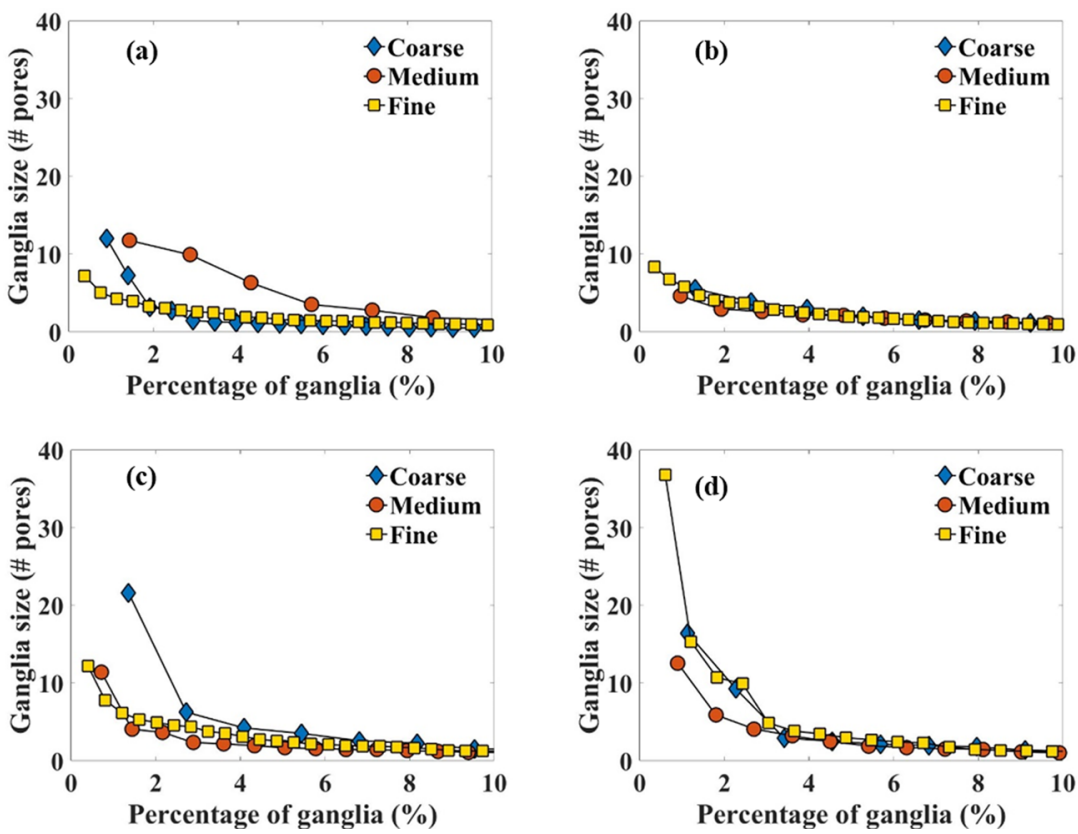

Figure 7. Size of trapped oil ganglia, displayed as the number of average sized pores filled in each micromodel at final conditions for experiments (a) $\mathrm{P}-\mathrm{O} 1,(\mathrm{~b}) \mathrm{P}-\mathrm{O} 2,(\mathrm{c}) \mathrm{W}-\mathrm{O} 1$, and (d) W-O2. Each point displays the percentage of the total ganglia of the given size.

441 number of ganglia are more likely to be controlled by the 442 morphology of the pore space.

443 If the same phenomena were to be reproduced on much 444 larger scales, more stable displacement produced by the 445 polymer means that the time until injectant breakthrough at 446 a production well would be increased in the case where 447 polymer is used. In oil fields this can have large economic 448 consequences in terms of well design, production rates, as well as environmental problems with produced water and reservoir 449 engineering issues once the injecting fluid has broken into the 450 wellbore (Dongo and Adewole ${ }^{34}$ ). Considering all these 451 adverse effects occur at breakthrough of the injecting fluid, it 452 makes sense that reservoir engineers aim to delay the 453 breakthrough time as much as possible. If these phenomena 454 exhibited on a small scale were to happen on a reservoir scale, 455 then the breakthrough time would be significantly delayed, 456 


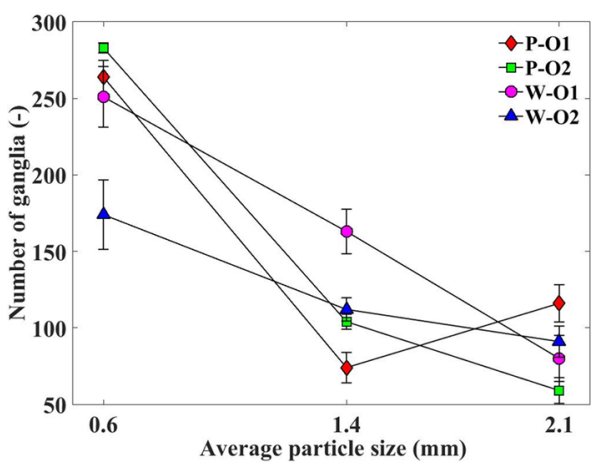

Figure 8. Final number of trapped oil ganglia as a function of average particle size at $T_{\mathrm{f}}$.

457 having a positive knock on effect on many other areas of 458 production.

\section{SUMMARY AND CONCLUSIONS}

459 Grain size is thought to have some effect on single-phase 460 permeability, but there have been few studies that consider the 461 effect on two-phase flow. In order to investigate this, a series of 462 displacement experiments were conducted using four immis463 cible fluid pairs using micromodels to investigate the effect of 464 pore and grain size on immiscible displacement on the 465 efficiency of polymer flooding. The model was initially 466 saturated with either Xiameter PMX - 200 Silicon Fluid 467 (Dow Corning, Midland, Michigan) or Exxsol D140 (Exxon 468 Mobil, Irving, Texas) which was then displaced by one of two 469 displacing fluids, either deionized water or xanthan solution $470(1000 \mathrm{ppm})$. The viscosity ratio $(\log M)$ ranged from -2.5 to 4710.84 and capillary number $(\log C a)$ from -4.6 to -2.3 . The 472 experiments were conducted using specially fabricated water473 wet micromodels, with images captured at $1 \mathrm{~s}$ intervals using an 474 optical microscope. These experiments extend the under475 standing of the effect of pore and grain size on immiscible 476 displacement during polymer flooding.

477 As expected, the polymer solution achieves a far higher oil 478 recovery in the majority of cases examined. The stability of the 479 displacement front was investigated both qualitatively and 480 quantitatively, with the xanthan solution producing more stable 481 displacement in comparison to water. The more stable 482 displacement front observed in the case of polymer solution 483 as the displacing fluid leads to a later breakthrough time. If this 484 is replicated at reservoir scales it could have significant 485 economic consequences as a result of this delayed displacing 486 fluid breakthrough into the wellbore.

487 Our results show that polymer flooding is less effective at 488 finer grain sizes, which in this experiment correspond to 489 medium-coarse grained sandstones. Because many reservoir 490 sandstones are fine to medium grained, ${ }^{32}$ this would suggest 491 that polymer flooding may not be more efficient than 492 waterflooding at improving recovery in many sandstones.

493 This study aimed to extend the knowledge of how polymer 494 flooding works at the pore-scale and provides insight into the 495 different scenarios in which polymer flooding is most effective. 496 The insights provided here will help to assess the suitability of a 497 reservoir for polymer flooding with regards to the pore sizes 498 found in the reservoir.

\section{ASSOCIATED CONTENT}

S Supporting Information

The Supporting Information is available free of charge on the 501 ACS Publications website at DOI: 10.1021/acs.energy- 502 fuels. $7 \mathrm{~b} 01254$.

Experimental setup (PDF)

\section{AUTHOR INFORMATION}

*School of Chemical Engineering and Analytical Science, 507 Room C26, The Mill, The University of Manchester, Sackville 508 Street, Manchester, M13 9PL, UK. Tel: 0441613063980. E- 509 mail: nima.shokri@manchester.ac.uk.

ORCID $\odot$

Nima Shokri: 0000-0001-6799-4888

Notes

The authors declare no competing financial interest.

\section{ACKNOWLEDGMENTS}

N.S. acknowledges the donors of the American Chemical 516 Society Petroleum Research Fund for partial support of this 517 research (PRF No. 52054-DNI6). We acknowledge the UK 518 Natural Environment Research Council (NERC) for providing 519 the Ph.D. studentship for M.L. The micromodel was fabricated 520 in the William R. Wiley Environmental Molecular Sciences 521 Laboratory, a U.S. Department of Energy scientific user facility 522 operated by Pacific Northwest National Laboratory.

523

\section{REFERENCES}

524

(1) Sahimi, M. Flow and Transport in Porous Media and Fractured 525 Rock, 2nd ed.; Wiley-VCH, 2012.

(2) Nilsson, M. A.; Kulkarni, R.; Gerberich, L.; Hammond, R.; Singh, 527 R.; Baumhoff, E.; Rothstein, J. P. Effect of Fluid Rheology on 528 Enhanced Oil Recovery in a Microfluidic Sandstone Device. J. Non- 529 Newtonian Fluid Mech. 2013, 202, 112.

530

(3) Wu, R.; Kharaghani, A.; Tsotsas, E. Two-phase flow with capillary 531 valve effect in porous media. Chem. Eng. Sci. 2016, 139, 241-248. 532

(4) Muggeridge, A.; Cockin, A.; Webb, K.; Frampton, H.; Collins, I.; 533 Moulds, T.; Salino, P. Recovery Rates, Enhanced Oil Recovery and 534 Technological Limits. Philos. Trans. R. Soc., A 2014, 372, 20120320. 535

(5) Zitha, P.; Felder, R.; Zornes, D.; Brown, K.; Mohanty, K. 536 Increasing Hydrocarbon Recovery Factors, SPE, 2014.

(6) Grassia, P.; Mas Hernandez, E.; Shokri, N.; Cox, S. J.; Mishuris, 538 G.; Rossen, W. R. Analysis of a Model for Foam Improved Oil 539 Recovery. J. Fluid Mech. 2014, 751, 346.

540

(7) Rabbani, H.; Joekar-Niasar, V.; Shokri, N. Effects of intermediate 541 wettability on entry capillary pressure in angular pores. J. Colloid 542 Interface Sci. 2016, 473, 34.

(8) Osei-Bonsu, K.; Shokri, N.; Grassia, P. Fundamental Investigation 544 of Foam Flow in a Liquid-Filled Hele-Shaw Cell. J. Colloid Interface Sci. 545 2016, 462, 288.

(9) Mas-Hernandez, E.; Grassia, P.; Shokri, N. Foam Improved Oil 547 Recovery: Modelling the effect of an increase in injection pressure. 548 Eur. Phys. J. E: Soft Matter Biol. Phys. 2015, 38, 67.

(10) Zhao, B.; MacMinn, C. W.; Juanes, R. Wettability control on 550 multiphase flow in patterned microfluidics. Proc. Natl. Acad. Sci. U. S. 551 A. 2016, 113, 10251.

(11) Rodríguez de Castro, A.; Oostrom, M.; Shokri, N. Effects of 553 Shear-thinning Fluids on Residual Oil Formation in Microfluidic Pore 554 Networks. J. Colloid Interface Sci. 2016, 472, 34. 555

(12) Al Sofi, A. M.; Blunt, M. J. Streamline-Based Simulation of Non- 556 Newtonian Polymer Flooding. SPE Journal 2010, 15, 901-911. 557 
558 (13) Lopez, X.; Valvatne, P. H.; Blunt, M. J. Predictive Network 559 Modelling of Single Phase Non-Newtonian Flow in Porous Media. J. 560 Colloid Interface Sci. 2003, 264 (1), 256.

561 (14) Emami Meybodi, H.; Kharrat, R.; Wang, X. Study of 562 Microscopic and macroscopy Displacement Behaviors of Polymer 563 Solution in Water Wet and Oil Wet media. Transp. Porous Media 2011, 56489 (1), 97.

565 (15) Buchgraber, M.; Clemens, T.; Castanier, L. M.; Kovscek, A. R. A 566 Microvisual Study of the Displacement of Viscous Oil by Polymer 567 Solutions. SPE Journal 2011, 14, 269.

568 (16) Sinton, D. Microfluidics and Their Macro Applications for the 569 Oil and Gas Industry. Way Ahead 2015, 11 (3), 8.

570 (17) Conn, C. A.; Ma, K.; Hirasaki, G. J.; Biswal, S. L. Visualising Oil 571 Displacement with Foam in a Microfluidic Device with Permeability 572 Contrast. Lab Chip 2014, 14, 3968-3977.

573 (18) Tahmasebi, P.; Sahimi, M.; Andrade, J. E. Image-based 574 Modelling of Granular Porous Media. Geophys. Res. Lett. 2017, 44, $5754738-4746$

576 (19) Tahmasebi, P.; Javadpour, F.; Sahimi, M. Three-Dimensional 577 Stochastic Characterization of Shale SEM Images. Transp. Porous 578 Media 2015, 110, 521-531.

579 (20) Weger, R. J.; Eberli, G. P.; Baechle, G. T.; Massaferro, J. L.; Sun, 580 Y.-F. Quantification of Pore Structure and Its Effect on Sonic Velocity 581 and Permeability in Carbonates. AAPG Bull. 2009, 93 (10), 1297.

582 (21) Rangel-German, E. R.; Kovscek, A. R. Experimental and 583 Analytical Study of Multidimensional Imbibition in Fractured Porous 584 Media. J. Pet. Sci. Eng. 2002, 36, 45.

585 (22) Rad, M. N.; Shokri, N.; Keshmiri, A.; Withers, P. J. Effects of 586 Grain and Pore Size on Salt Precipitation During Evaporation from 587 Porous Media: A pore-scale investigation. Transp. Porous Media 2015, 588110 (2), 281.

589 (23) Grate, J. W.; Dehoff, K. J.; Warner, M. G.; Pittman, J. W.; 590 Wietsma, T. W.; Zhang, C.; Oostrom, M. Correlation of oil-water and 591 air-water contact angles of diverse silanized surfaces and relationship to 592 fluid interfacial tensions. Langmuir 2012, 28, 7182.

593 (24) Willingham, T.; Zhang, C.; Werth, C.; Valocchi, A. J.; Oostrom, 594 M.; Wietsma, T. W. Using Dispersivity Values to Quantify the Effects 595 of Pore-Scale Flow Focusing on Enhanced Reaction Along a 596 Transverse Mixing Zone. Adv. Water Resour. 2010, 33, 525.

597 (25) Rabbani, H. S.; Joekar-Niasar, V.; Pak, T.; Shokri, N. New 598 insights on the complex dynamics of two-phase flow in porous media 599 under intermediate-wet conditions. Sci. Rep. 2017, 7, 4584.

600 (26) Kern, W. The Evolution of Silicon Wafer Cleaning Technology. $601 \mathrm{~J}$. Electrochem. Soc. 1990, 137, 1887.

602 (27) Lenormand, R.; Zarcone, C.; Sarr, A. Mechanisms of the 603 Displacement of One Fluid by Another in a Network of Capillary 604 Ducts. J. Fluid Mech. 1983, 135, 337.

605 (28) Bandara, U. C.; Oostrom, M.; Tartakovsky, A. M.; Palmer, B. J.; 606 Zhang, C.; Grate, J. W. Comparison of pore-scale numerical 607 simulations of unstable immiscible displacements in porous media 608 with micromodel experiments. Adv. Water Resour. 2013, 62, 356-369. 609 (29) Zhang, C. Y.; Oostrom, M.; Wietsma, T. W.; Grate, J. W.; 610 Warner, M. G. Viscous and capillary effects on immiscible fluids 611 displacement: pore-scale study in a uniform pore network micromodel. 612 Energy Fuels 2011, 25 (8), 3493-3505.

613 (30) Herbas, J. G.; Wegner, J.; Hincapie, R. E.; Födisch, H.; Ganzer, $614 \mathrm{~L}$. Comprehensive Micromodel Study to Evaluate Polymer EOR in 615 Unconsolidated Sand Reservoirs; Society of Petroleum Engineers, 2011; 616 SPE-172669-MS.

617 (31) Lenormand, R.; Touboul, E.; Zarcone, C. Numerical Models 618 and Experiments on Immiscible Displacements in Porous Media. J. 619 Fluid Mech. 1988, 189, 165.

620 (32) Bloch, S.; McGowan, J. Influence of Depositional Environment 621 on Reservoir Quality Prediction. In Reservoir Quality Assessment and 622 Prediction in Clastic Rocks; Wilson, M., Ed.; Society for Sedimentary 623 Geology: Tulsa, OK, 1994; Vol. 30.

624 (33) Payatakes, A. C. Dynamics of Oil Ganglia During Immiscible 625 Displacement in Water-Wet Porous Media. Annu. Rev. Fluid Mech. $6261982,14,365$.
(34) Dongo, V. E.; Adewole, E. S. Factors Affecting Water 627 Breakthrough Time of a Vertical Well Subject to Double-Edge 628 Water Drive Mechanism. Int. J. Eng. Res. Afr. 2014, 11, 51. 